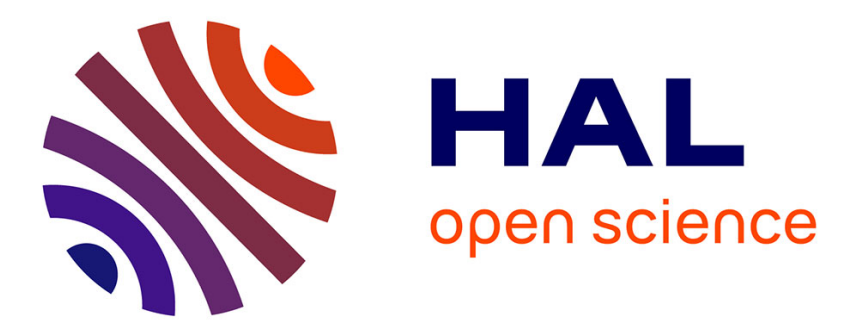

\title{
On the microwave breakdown stability of a spherical hot spot in air
}

\author{
J Rasch, V E Semenov, D Anderson, M Lisak, J Puech
}

\section{To cite this version:}

J Rasch, V E Semenov, D Anderson, M Lisak, J Puech. On the microwave breakdown stability of a spherical hot spot in air. Journal of Physics D: Applied Physics, 2010, 43 (32), pp.325204. 10.1088/0022-3727/43/32/325204 . hal-00569670

\section{HAL Id: hal-00569670 \\ https://hal.science/hal-00569670}

Submitted on 25 Feb 2011

HAL is a multi-disciplinary open access archive for the deposit and dissemination of scientific research documents, whether they are published or not. The documents may come from teaching and research institutions in France or abroad, or from public or private research centers.
L'archive ouverte pluridisciplinaire HAL, est destinée au dépôt et à la diffusion de documents scientifiques de niveau recherche, publiés ou non, émanant des établissements d'enseignement et de recherche français ou étrangers, des laboratoires publics ou privés. 


\title{
On the Microwave Breakdown Stability of a Spherical Hot Spot
} in Air

\author{
J. Rasch ${ }^{1}$, V. E. Semenov², D. Anderson ${ }^{1}$, M. Lisak ${ }^{1}$ and J. Puech ${ }^{3}$ \\ ${ }^{1}$ Chalmers University of Technology, Göteborg, Sweden \\ 2 Institute of Applied Physics, Nizhny Novgorod, Russia \\ ${ }^{3}$ Centre National d'Études Spatiales, 31401 Toulouse Cedex 9, France
}

\begin{abstract}
An analysis is made of the basic physical conditions under which a small local microwave induced breakdown region in a gas may develop to extended "global" breakdown. The analysis describes the different nonlinear stages of the microwave breakdown process. In the first stage, the increasing breakdown plasma density suppresses the electric field in the breakdown region to reach a quasistationary state with constant electron density. The subsequent heating of the gas due to absorption of microwave power in the breakdown plasma is then analyzed and the corresponding steady state for the thermal evolution is found including the temperature dependence of the breakdown electric field. The stability properties of the stationary state are examined and it is found that there exists a critical (unstable) radius of the initial breakdown plasma region such that initial regions smaller than this critical dimension will shrink to ultimately vanish whereas plasma regions larger than the critical dimension will grow indefinitely and transform the local breakdown region into full scale "global" breakdown. The practical implication of this model is to give an order of magnitude estimate for the critical size of hot spots, regions of enhanced field and intensified heating in rf systems.
\end{abstract}




\section{INTRODUCTION}

Microwave breakdown of gases has for a long time been recognized as an important failure mechanism in microwave systems. The general physical mechanisms underlying the phenomenon are well understood ${ }^{1,2}$ - free electrons are heated by the microwave electric field and a fraction of these electrons (in the tail of the electron energy distribution) will acquire sufficient energy to make ionizing impacts with neutral gas particles. If the electric field is strong enough, the number of free electrons increases exponentially, i.e. breakdown occurs. The actual value of the electric field at which breakdown is initiated is determined by the condition that the ionization frequency balances the two dominant loss mechanisms of free electrons, attachment to neutral molecules and diffusion out of the breakdown region. Both these latter mechanisms can be considered as independent of the electric field in comparison with the ionization frequency, which depends strongly on the field strength. When the number of free electrons in a gas increases, the gas eventually becomes conducting, which changes the general properties of the microwave system and may even cause serious damage.

Although many studies have been made of the conditions under which microwave breakdown occurs in particular geometries and of the specific breakdown thresholds related to these systems e.g. ${ }^{1,3}$, much less attention has been given to studies of the post-breakdown (nonlinear) evolution of the breakdown plasma. This problem is especially important in microwave devices involving strongly inhomogeneous fields, since edges and similar features in conducting parts of a device will lead to strong local field enhancements ${ }^{4}$. It is well known that a breakdown plasma may form in these high field regions even for low ambient fields, but the subsequent effects of such small regions of plasma formation are not well understood. Experimentally it is often observed that plasma regions tend to absorb microwave power, thus heating the gas as well as neighboring parts of the device walls, even to the point of melting sharp details like screws and soldering points. It is also well known that heating of the gas tends to lower the breakdown threshold ${ }^{5,6}$. Consequently, a region of high temperature is potentially dangerous as a starting point for breakdown development into full scale breakdown. A natural and fundamental question for this type of situation is whether a small plasma region, created e.g. by a local enhancement of the electric field, will produce enough heat to instigate breakdown in the main parts of the system, i.e. a "global" breakdown? It can be intuitively understood that if the field enhancement is high 
and the region is small, the heat produced by the plasma will not be sufficient to make the breakdown region expand. On the other hand, if the difference in field strength between the local high field region and the ambient "global" electric field is small and the high field region is large enough, there should be a transition from local to global breakdown.

Even though it might be possible to take into account all relevant physical processes in a computer simulation, the concomitantly gained physical understanding would be limited. In the present work we will use an alternative approach by starting with a simple model that takes into account only the most important physical effects. Clearly, this implies that the direct practical applicability of the model will be restricted, but it will make it possible to analyze and understand the interplay between the different physical processes involved in the problem and to indicate proper directions for further investigations.

The present analysis will consider as initial condition a homogeneous microwave electric field in Air at room temperature and also containing a spherical region of high temperature - a "hot spot". The gas pressure is assumed to be high enough so that electron losses are dominated by attachment and the dimension of the hot spot region is assumed to be smaller than the wavelength, so that the quasi-static approximation can be used for describing the self-consistent electric field in the hot spot region. The electric field strength is below the breakdown threshold of the low temperature gas, but above the threshold of the hot spot region. The initial development of the breakdown process is obvious: the electron density in the hot spot will rapidly increase until the density is large enough to suppress the electric field down to the breakdown value corresponding to the high temperature. The subsequent evolution constitutes a problem of thermal stability, the breakdown plasma will be heated by absorption of the electric field and if the plasma sphere is larger than a certain radius, the heat generated in the plasma region will be larger than the heat transported out of the plasma by thermal conduction. Under such circumstances, the plasma ball will expand indefinitely. On the other hand, if the radius is smaller than the critical value, the plasma will absorb less heat than what is lost and will shrink monotonously until it disappears completely. The solution of this model problem provides important practical information by determining the order of magnitude of the spatial scale when a localized hot spot region becomes prone to global breakdown in a mainly homogeneous field. 


\section{BASIC BREAKDOWN PHYSICS}

The problem under consideration involves several different physical processes: the heating of the free electrons, the increasing free electron density due to the ionizing action of the electric field, the local suppression of the electric field by the increasing electron density, the heating of the neutral particles by collisions with electrons, the conduction of heat out of the critical region and finally the expansion or contraction of the plasma region. An important property of the dynamics of this problem is the fact that the time scales for these different processes are very different, a feature which makes possible an analytically tractable solution.

When a microwave electric field is applied to a gas with a low density of free electrons, these electrons are rapidly heated in a time of the order of $\left(\delta_{\text {coll }} \nu_{c}\right)^{-1} \mathrm{~s}$, where $\delta_{\text {coll }}$ is the relative fraction of energy lost in the collision of an electron with a neutral and $\nu_{c}$ is the corresponding collision frequency. Since typically in Air $\delta_{\text {coll }} \approx 10^{-2}$ and $\nu_{c} \approx 5 \cdot 10^{9} p 1 / \mathrm{s}$ ( $p$ measured in Torr), this time scale is very short, e.g. of the order of $10^{-10} \mathrm{~s}$ for pressures $p \approx 200 \operatorname{Torr}^{2,5}$. The heated electrons will then ionize the neutrals and make the electron density increase exponentially on a time-scale that can be estimated as being of the order of the attachment time scale i.e. $\left(\nu_{a}\right)^{-1} \approx 10^{-8} \mathrm{~s}$, where $\nu_{a} \approx 6.4 \cdot 10^{4} p 1 / \mathrm{s}$ ( $p$ in Torr $)$ is the frequency for electron losses by attachment on neutrals ${ }^{3,7}$. The subsequent heating of the neutral Air particles is a very slow process until the electron density becomes high, since it depends on the ratio of electron to neutral densities. The presence of such clearly separated time scales makes it possible to consider the electron energy and consequently also the ionization and attachment frequencies as determined by the average local temperature of the neutral gas.

The problem also involves well separated spatial length scales. The inherent length scale of the microwave field is the wavelength, $\lambda$, which is typically a few centimeters at $\mathrm{GHz}$ frequencies. The dimension of the considered hot spot region, $L_{p}$, is assumed to be significantly smaller than $\lambda$. On the other hand, a third length scale is determined by the extension of the transition region between the plasma in the hot spot and the surrounding gas. The dimension of this transition region can be estimated as the attachment length given by $L_{a}=\sqrt{D_{a} / \nu_{a}}$, where $D_{a} \approx 1.4 \cdot 10^{4} / p \mathrm{~cm}^{2} / \mathrm{s}$ ( $p$ in Torr) is the ambipolar diffusion coefficient, which implies that $L_{a} \approx 10^{-3} \mathrm{~cm}$ at atmospheric pressures ${ }^{5}$. This length provides 
a lower bound for the size of the plasma region. Thus, the subsequent analysis is valid provided the following relation between the length scales is satisfied: $L_{a} \ll L_{p} \ll \lambda$.

When the breakdown plasma has formed under the ionizing action of the electric field, the further development of the breakdown process becomes non-linear since the electric field strength inside the plasma is suppressed, which affects the ionization and constitutes a feed back mechanism that self-consistently determines the extension of the plasma configuration. Since the dimension of the hot spot is much smaller than the wavelength, the quasi-static approximation can be used to describe the electric field. This means that the plasma can be regarded as a dielectric which affects the applied electric field and alters its strength. The dielectric function in a weakly ionized gas is given by

$$
\epsilon=1-\frac{N e^{2}}{\epsilon_{0} m\left(\omega^{2}+\nu_{c}^{2}\right)}-i \frac{\nu_{c}}{\omega} \frac{N e^{2}}{\epsilon_{0} m\left(\omega^{2}+\nu_{c}^{2}\right)}
$$

where $N$ is the electron density and $\omega$ is the microwave frequency. Defining $n=$ $N e^{2} /\left(\epsilon_{0} m\left(\omega^{2}+\nu_{c}^{2}\right)\right)$, and $\delta=\nu_{c} / \omega$, the above expression can be written as

$$
\epsilon=1-n-i n \delta
$$

Under the above assumptions and using the fact that $\nabla \cdot(\epsilon \mathbf{E})=0$, the electric field strength inside a spherical region of plasma in a homogeneous field is suppressed down to the value ${ }^{8}$

$$
E=\left|\frac{3 E_{0}}{\epsilon+2}\right|=\frac{3 E_{0}}{\sqrt{(3-n)^{2}+\delta^{2} n^{2}}}
$$

which is equal to the breakdown electric field corresponding to the high temperature region. After the rapid establishing of the breakdown plasma and the associated quasi-stationary state, the plasma starts to absorb energy from the microwave field by Joule heating at a rate given by $W=\mathbf{j} \cdot \mathbf{E}=-N e \mathbf{v} \cdot \mathbf{E}$ where the electron velocity, $\mathbf{v}$, is determined by

$$
m \frac{d \mathbf{v}}{d t}=-e \mathbf{E}-m \nu_{c} \mathbf{v}
$$

which for a sinusoidally varying electric field, $\mathbf{E}=\mathbf{E}_{\mathbf{0}} \cos (\omega t)$, becomes

$$
\mathbf{v}=\frac{-e \mathbf{E}_{\mathbf{0}}}{m\left(\omega^{2}+\nu_{c}^{2}\right)}\left(\nu_{c} \cos (\omega t)+\omega \sin (\omega t)\right)
$$

This means that the averaged absorption rate is

$$
q_{p} \equiv\langle W\rangle=\frac{\omega}{2 \pi} \int_{0}^{\frac{2 \pi}{\omega}} W d t=\frac{1}{2} E_{0}^{2} \frac{N e^{2} \nu_{c}}{m\left(\omega^{2}+\nu_{c}^{2}\right)}=\epsilon_{0} \omega n \delta E_{r m s}^{2}
$$

where from now on the subscript rms will be dropped and $E$ will be used to denote the root-mean-square electric field strength. 


\section{BREAKDOWN AND PLASMA HEATING}

In an analysis of the development of the breakdown process, the first step involves the fast ionization process where the electron density increases. This evolution is governed by the continuity equation for the electron density

$$
\frac{\partial N}{\partial t}=\nabla^{2}\left(D_{a} N\right)+\left(\nu_{i}-\nu_{a}\right) N-\alpha N^{2}
$$

where the last term accounts for recombination losses. As discussed above, diffusion losses are small under the present assumption of high gas pressure, and can be neglected. Furthermore, the recombination frequency $\alpha N$ becomes important only when $N \approx \nu_{a} / \alpha \approx$ $6.4 \cdot 10^{4} \mathrm{p} / 10^{-7} \approx 10^{14} \mathrm{~cm}^{-3}$. However, as will be demonstrated below, the plasma density rapidly saturates at a level $n \sim \delta^{-1}$, implying $N \sim 10^{12} \mathrm{~cm}^{-3}$ for microwave frequencies in the $\mathrm{GHz}$ range. Thus, the recombination losses can also be neglected and the remaining continuity equation becomes simply

$$
\frac{\partial N}{\partial t}=N\left(\nu_{i}-\nu_{a}\right)
$$

The basic physical mechanism in the first stage of the nonlinear evolution is that the electron density increases until the local electric field is suppressed by the dielectric property of the plasma to become equal to the breakdown field in the heated region, i.e. $E=E_{a} \Leftrightarrow \nu_{i}(E)=$ $\nu_{a}$. Thus, a steady state is reached where the equality $\nu_{i} \approx \nu_{a}$ is satisfied or correspondingly for the electric field

$$
E_{a}=E=\frac{3 E_{0}}{\sqrt{(3-n)^{2}+\delta^{2} n^{2}}}
$$

For the case relevant here, i.e. $\delta \gg 1$, the modified field varies from $E=E_{0}$ at $n=0$ to rapidly reach the asymptotic monotonous decrease given by $E \approx 3 E_{0} /(\delta n)$. The general features of the variation of $E_{a}$ with $n$ in the case when $\delta \gg 1$ are shown in Fig.1. The electron density necessary to suppress the field to the level $E_{a}$ is given by

$$
n=\frac{3}{\delta^{2}}\left[1+\sqrt{\left(\left(\frac{E_{0}}{E_{a}}\right)^{2}-1\right) \delta^{2}+1}\right]
$$

The remaining problem is a purely thermal one since the electric field can be considered as adiabatically following the temperature so that the corresponding breakdown level is preserved. The heating rate becomes

$$
q_{p}=\epsilon_{0} \omega n \delta E_{a}^{2}=\frac{3 \epsilon_{0} \omega E_{a}^{2}}{\delta}\left[1+\sqrt{\left(\left(\frac{E_{0}}{E_{a}}\right)^{2}-1\right) \delta^{2}+1}\right]
$$




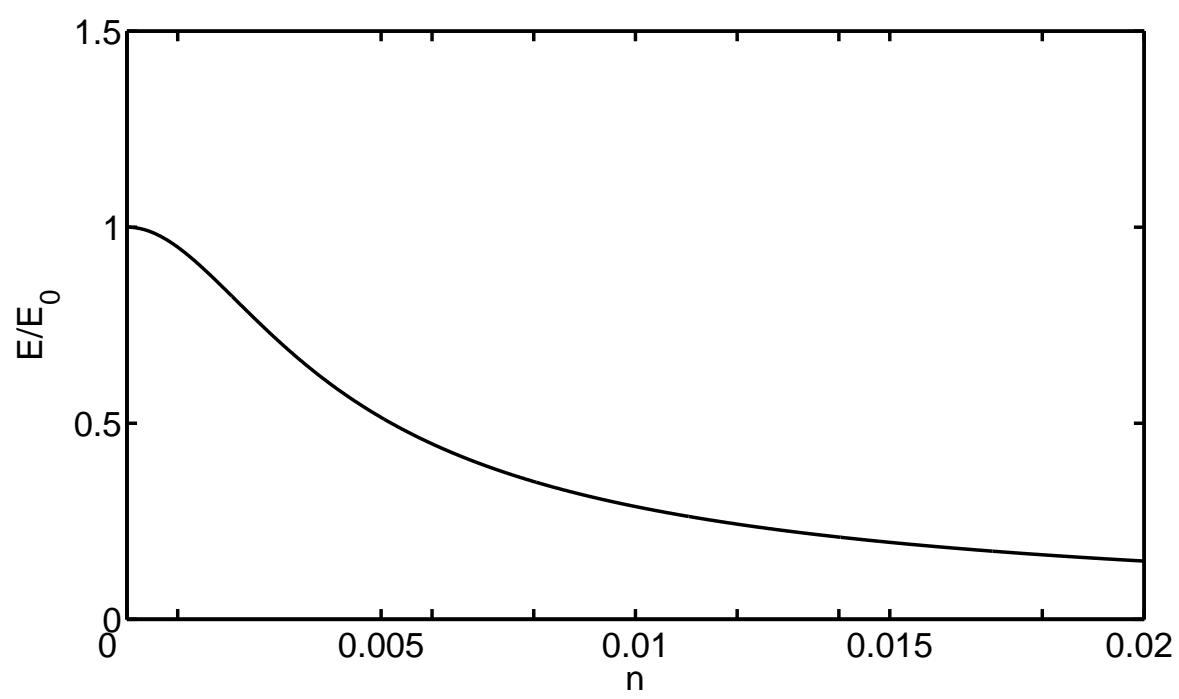

FIG. 1: The local field as a function of electron density for $\delta=1000$.

In order to rewrite the heating rate as a function of temperature we note that in a large range of (high) pressures $E_{a} \propto p$. However, the crucial parameter determining the breakdown strength is not the gas pressure, but rather the gas density, and an increase in temperature under constant pressure leads to a decreasing neutral gas density ${ }^{5}$. This implies that the dependence of the suppressed field on temperature can be written as $E_{a} \approx E_{a 0} T_{0} / T$. Similarly we note that $\delta=\nu_{c} / \omega$ where $\nu_{c} \propto p$, which implies that $\delta \approx \delta_{0} T_{0} / T$.

Using these temperature dependencies, the heating term can be expressed as

$$
q_{p}=\frac{3 \epsilon_{0} \omega E_{a 0}^{2}}{\delta_{0}} \frac{T_{0}}{T}\left[1+\sqrt{\left(\left(\frac{E_{0}}{E_{a 0}}\right)^{2}-\left(\frac{T_{0}}{T}\right)^{2}\right) \delta_{0}^{2}+1}\right]
$$

For $T<T_{1} \equiv T_{0} \frac{E_{a 0}}{E_{0}}$ the microwave field is below the breakdown threshold, implying that no breakdown plasma exists and consequently no heating occurs i.e. $q_{p}=0$. When $T=T_{1}$, the heating term is very small, but when $T$ increases the heating will rapidly rise to high values, and we can neglect the two terms of order unity in the parenthesis. The approximate expression for the plasma heating term becomes

$$
q_{p}\left(T>T_{1}\right)=3 \epsilon_{0} \omega E_{0}^{2} \frac{T_{1}}{T} \sqrt{1-\left(\frac{T_{1}}{T}\right)^{2}}
$$

Introducing the parameter $q_{E} \equiv 3 \epsilon_{0} \omega E_{0}^{2}$, which only depends on the electric parameters of the system, the heating term can be written in a simple form that emphasizes the temper- 
ature dependence viz

$$
q_{p}=q_{E} \frac{T_{1}}{T} \sqrt{1-\left(\frac{T_{1}}{T}\right)^{2}}
$$

The heating term vanishes for $T<T_{1}$, rapidly increases when $T$ exceeds $T_{1}$ to reach the maximum value $q_{m}=q_{E} / 2$ at the temperature $T / T_{1}=\sqrt{2}$ and then decays monotonously. The features of this dependence is illustrated in Fig.2,

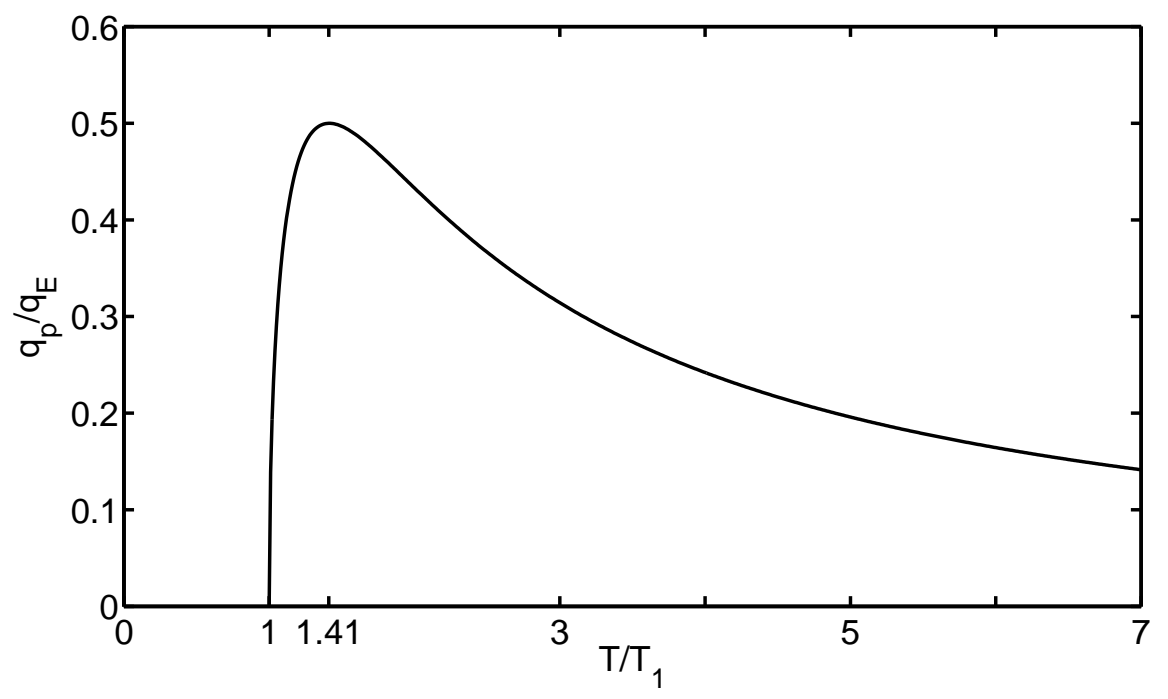

FIG. 2: The normalized heating term as a function of $T / T_{1}$.

\section{THERMAL EQUILIBRIUM}

In the previous section an expression was derived for the gas heating due to microwave absorption in the breakdown plasma. The remaining task is a purely thermal one - to determine the evolution of the Air temperature in the hot spot plasma, other relevant physical quantities being determined by the temperature. Under the assumptions of relatively low temperatures (c.f. below) and small space scales, the corresponding heat equation can be written

$$
\rho c_{v} \frac{\partial T}{\partial t}=\nabla(\kappa \nabla T)+q
$$

where $\rho$ denotes the density, $c_{v}$ is the heat capacity and $\kappa$ is the thermal conductivity. Of primary interest is the equilibrium situation described by the two coupled equations

$$
\begin{array}{ll}
\nabla(\kappa \nabla T)+q(T)=0, & r<R \\
\nabla(\kappa \nabla T)=0, & r>R
\end{array}
$$


These equations allow the trivial (and harmless) solution $T=$ constant $<T_{1}$, which actually represents the standard situation in electrical systems. In order to determine other possible solutions involving regions $T>T_{1}$, it is necessary to know the dependence of the thermal conductivity, $\kappa$, on $T$. The specific form of $\kappa$ depends on the type of gas and on the appropriate temperature interval under consideration, but can often be approximated as a power law dependence, $\kappa \approx \kappa_{0}\left(T / T_{0}\right)^{\beta}$, e.g. a good approximation of the thermal conductivity of Air for temperatures less than $2000 \mathrm{~K}$ is obtained for $\beta=3 / 4$, c.f. ${ }^{6,9}$. Because of the spherical symmetry, the heat equation in the region outside the hot spot is

$$
\frac{1}{r^{2}} \frac{\partial}{\partial r}\left(r^{2} \kappa_{0}\left(\frac{T}{T_{0}}\right)^{\beta} \frac{\partial T}{\partial r}\right)=0
$$

Using the boundary conditions $T(R)=T_{1}$ and $T(r \rightarrow \infty)=T_{0}$ this equation is easily solved as

$$
T^{\beta+1}(r>R)=\frac{R}{r}\left(T_{1}^{\beta+1}-T_{0}^{\beta+1}\right)+T_{0}^{\beta+1}
$$

and consequently

$$
\left.\frac{\partial T}{\partial r}\right|_{r>R}=-\frac{R}{r^{2}} \frac{\left(T_{1}^{\beta+1}-T_{0}^{\beta+1}\right)}{(\beta+1) T^{\beta}}
$$

In particular, the derivative at $r=R$ is

$$
\left.\frac{\partial T}{\partial r}\right|_{r=R}=\frac{T_{0}\left(\frac{T_{0}}{T_{1}}\right)^{\beta}-T_{1}}{(\beta+1) R}
$$

The corresponding equation in the hot spot is more difficult to solve, but a good approximate solution can be obtained by integrating Eq.(16) over the spherical volume $r \leq R$ to obtain

$$
4 \pi \int \frac{1}{r^{2}} \frac{\partial}{\partial r}\left(\kappa(T) r^{2} \frac{\partial T}{\partial r}\right) r^{2} d r+4 \pi \int q_{p}(T) r^{2} d r=0
$$

The volume integral of $q_{p}$ can approximately be evaluated as $4 \pi R^{3} q_{m} / 3$, where $q_{m}$ is the maximum value of the heating term, i.e. $q_{m}=q_{E} / 2$. On the boundary at $r=R$ this implies

$$
\left.R^{2} \kappa(T) \frac{\partial T}{\partial r}\right|_{r=R}+\frac{q_{m} R^{3}}{3}=0
$$

Together with the previous result for the temperature gradient at $r=R$, this gives

$$
\frac{\kappa\left(T_{1}\right)}{\beta+1}\left(T_{1}-T_{0}\left(\frac{T_{0}}{T_{1}}\right)^{\beta}\right)=\frac{q_{m} R^{2}}{3}
$$

Formally we have two solutions $R= \pm R_{0}$, but only the positive one represents a physical situation. We will later see that the result obtained in Eq.(23) is a good approximation and for easier comparison it can be rewritten in the more explicit form

$$
6\left(1-\left(\frac{T_{0}}{T_{1}}\right)^{\beta+1}\right)=\frac{(\beta+1) q_{E} R^{2}}{T_{1} \kappa\left(T_{1}\right)} \equiv \Lambda
$$




\section{NUMERICAL SOLUTION OF THE HEAT EQUATION}

To complement and assess the previous approximate solution, a numerical solution is found for the heat equation in spherical geometry i.e.

$$
\begin{array}{ll}
\frac{1}{r^{2}} \frac{\partial}{\partial r}\left[r^{2} \kappa_{1}\left(\frac{T}{T_{1}}\right)^{\beta} \frac{\partial T}{\partial r}\right]+q_{E} \frac{T_{1}}{T} \sqrt{1-\left(\frac{T_{1}}{T}\right)^{2}}=0, & T>T_{1} \\
\frac{1}{r^{2}} \frac{\partial}{\partial r}\left[r^{2} \kappa_{1}\left(\frac{T}{T_{1}}\right)^{\beta} \frac{\partial T}{\partial r}\right]=0, & T<T_{1}
\end{array}
$$

Where $\kappa_{1}=\kappa\left(T_{1}\right)=\kappa_{0}\left(\frac{T_{1}}{T_{0}}\right)^{\beta}$. It is convenient to write the equation in normalized variables by introducing $\Psi=\left(\frac{T}{T_{1}}\right)^{\beta+1}$ and $r=R x$ :

$$
\begin{array}{ll}
\frac{1}{x^{2}} \frac{\partial}{\partial x}\left(x^{2} \frac{\partial \Psi}{\partial x}\right)+\Lambda \Psi^{-1 /(\beta+1)} \sqrt{1-\Psi^{-2 /(\beta+1)}}=0, & \Psi>1 \\
\frac{1}{x^{2}} \frac{\partial}{\partial x}\left(x^{2} \frac{\partial \Psi}{\partial x}\right)=0, & \Psi<1
\end{array}
$$

where $\Lambda=\frac{(\beta+1) q_{E} R^{2}}{\kappa_{1} T_{1}}$ plays the role of an eigenvalue. Using the facts that $\Psi(x \rightarrow \infty) \rightarrow \Psi_{0}=$ $\left(\frac{T_{0}}{T_{1}}\right)^{\beta+1}$ and $\Psi(1)=1$, the derivative at $x=1$ is obtained directly as $\partial \Psi(1) / \partial x=\Psi_{0}-1$. It then remains only to solve the equation for $x<1$ with the appropriate boundary conditions i.e. the system of equations reduces to

$$
\begin{aligned}
& \frac{1}{x^{2}} \frac{\partial}{\partial x}\left(x^{2} \frac{\partial \Psi}{\partial x}\right)+\Lambda \Psi^{-1 /(\beta+1)} \sqrt{1-\Psi^{-2 /(\beta+1)}}=0, \quad x<1 \\
& \Psi(1)=1 \\
& \frac{\partial \Psi}{\partial x} \mid x=1=\Psi_{0}-1
\end{aligned}
$$

The solution of this nonlinear eigenvalue problem determines $\Lambda$ as a function of $\Psi_{0}<1$ for given value of $\beta$. The numerical solution of Eq.(29) is performed using the Euler method, and the result is shown in Fig.3 for some representative values of $\beta$. Plotted is also the approximate expression for the eigenvalue given by Eq.(24). The comparison shows that this simple expression is a reasonable approximation for values of $\beta$ smaller than unity, which implies that it provides a good approximation for Air (where $\beta \approx 3 / 4$ ).

The numerical solution also makes it possible to check the simplifying approximation of constant temperature in the hot spot region made in the analytical analysis. Fig. 4 shows the actual variation of the temperature in the breakdown region for some values of $\Psi_{0}$. Clearly, for reasonably small values of $\Psi_{0}$, the temperature variation in the breakdown region is not very large and the approximation as given by Eq.(24) should provide a good estimate of the critical radius. 


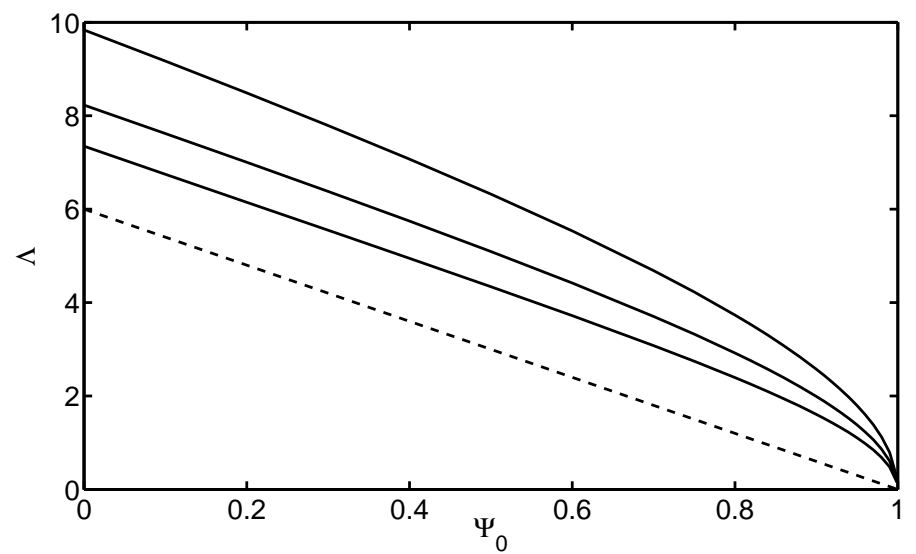

FIG. 3: The eigenvalue $\Lambda$ as a function of $\Psi_{0}$ for $\beta=[2,3 / 4,0.1]$ (from top to bottom). The dashed line represents the approximate solution given by Eq.(24).

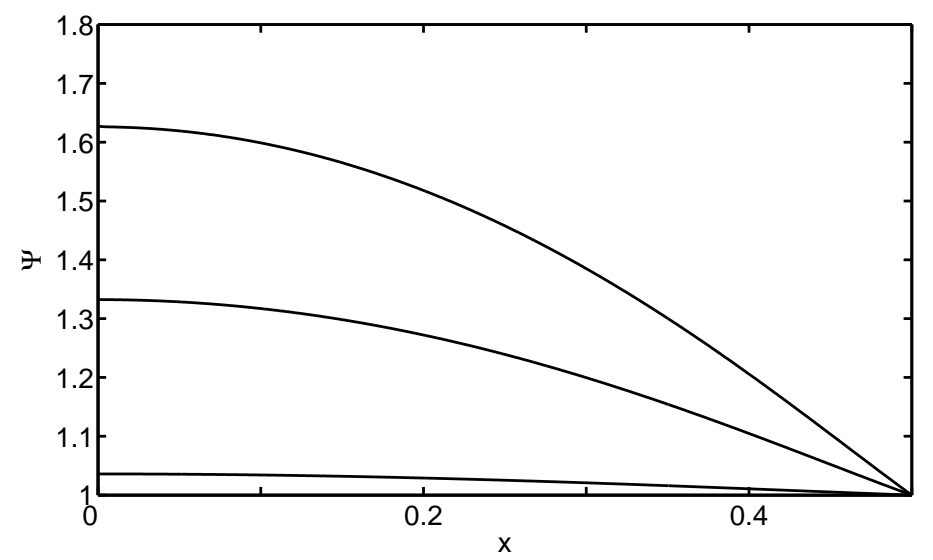

FIG. 4: The temperature profile for $\Psi_{0}=[0,0.5,0.95]$ from top to bottom.

\section{STABILITY}

In the previous section two physically relevant stationary solutions to the system of heat equations were found - the homogeneous non-breakdown solution, and the inhomogeneous solution corresponding to the self consistent balance between heating of the gas by absorption of microwave power and losses due to thermal conduction. A subsequent important problem is the stability properties of the latter solution.

The total heat equation reads

$$
\frac{\partial}{\partial t}\left(\rho c_{v} T\right)=\nabla(\kappa \nabla T)+q(T)
$$

Integration of this equation over the volume of the hot spot gives a relation that can be 
written as

$$
\frac{\partial W_{\text {total }}}{\partial t}=Q_{\text {loss }}+Q_{\text {Joule }}
$$

where $W_{\text {total }}$ represents the total heat content in the breakdown volume. $Q_{\text {loss }}$ denotes the total heat flux over the boundary of this region and can be written as

$$
Q_{\text {loss }}=4 \pi \kappa_{1} \frac{T_{0}\left(\frac{T_{0}}{T_{1}}\right)^{\beta}-T_{1}}{\beta+1} R
$$

Finally, $Q_{J o u l e}$ is the total microwave power absorbed in the breakdown region, a quantity which can be approximated as (c.f. the discussion in section IV.)

$$
Q_{\text {Joule }}=4 \pi \frac{q_{m}}{3} R^{3}
$$

The stationary situation discussed in the previous section corresponds to the case when the heat loss balances the heat generation. The total heat content of the breakdown region then remains constant and the temperature at the edge of the breakdown region is maintained. The stability of this configuration can be directly inferred by considering the dependence of the heating and loss terms on the radius $R$ of the breakdown region as shown in Fig.5.

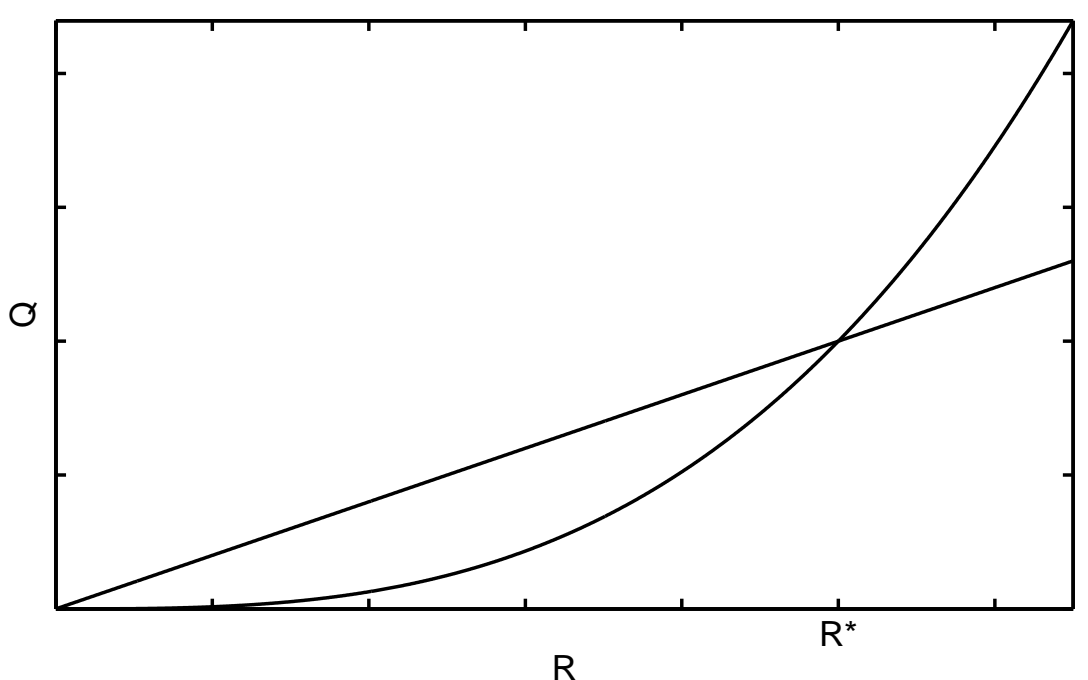

FIG. 5: The heat loss (straight line) and absorbed power (curved line) as functions of $R$.

It is clear from Fig.5 that there are two points of equilibrium; $R=0$ and $R=R^{*}$. The solution $R=0$ is clearly stable whereas the $R^{*}$ solution is unstable since a decrease in radius will lead to a situation where the heat loss is larger than the heat generation, which means that the temperature on the plasma edge cannot be maintained and the plasma will 
shrink until it eventually reaches the other stationary solution $R=0$, i.e. the breakdown region disappears. On the other hand, if there is an increase in radius from $R^{*}$, the opposite situation occurs i.e. the breakdown region will absorb more heat power than it can transport out over its boundary and the size of the breakdown plasma will consequently increase monotonously.

\section{CRITICAL RADIUS FOR AIR}

The previous analysis has established that there is a critical radius for a hot spot region with concomitant plasma formation in a homogeneous electric field in a gas where attachment is the dominating electron loss mechanism. The critical radius can be obtained by putting the left hand side of Eq.(31) equal to zero, which gives

$$
R=\sqrt{\frac{2 \kappa_{0} T_{0}}{\epsilon_{0}(\beta+1)} \frac{\left(\eta^{\beta+1}-1\right) \eta^{2}}{\omega E_{a 0}^{2}}}
$$

Where $\eta \equiv E_{a 0} / E_{0}$. The heat conductivity of Air can be approximated as ${ }^{6,9}$

$$
\kappa(T) \approx 6.15 \cdot 10^{-4}\left(\frac{T}{1000 \mathrm{~K}}\right)^{3 / 4} \quad\left(\frac{\mathrm{W}}{\mathrm{cm} \cdot \mathrm{K}}\right)
$$

which is a reasonable approximation for temperatures less than $2000 \mathrm{~K}$. When the temperature increases beyond $2000 \mathrm{~K}$, substantial production of NO molecules occurs. These molecules have a lower threshold for ionization than $\mathrm{O}_{2}$ and will eventually dominate the ionization process ${ }^{10}$. Thus, $T=2000 \mathrm{~K}$ is the upper limit for allowable temperatures in order for the model to be applicable in Air. The maximum temperature for different $\Psi_{0} \equiv\left(T_{0} / T_{1}\right)^{\beta+1}$ was shown in Fig.4 and indicates that the maximum temperature is approximately $1.3 T_{1}$. Thus, the model will not be valid if $T_{1}$ is larger than approximately $1550 \mathrm{~K}$. Breakdown is achieved at $T=T_{1}=T_{0} E_{a 0} / E_{0}=T_{0} \eta$ and assuming room temperature, i.e. $T_{0} \approx 300 \mathrm{~K}$,

the maximum allowed value of $\eta$ is about 5. Using the well known approximation for Air, $E_{a} / p \approx 30(\mathrm{~V} / \mathrm{cm}$ Torr $)$, one can calculate the critical radius, or rather the product $R^{*} \sqrt{\omega}$, as a function of $\eta$. The result is shown in Fig.6 below, in the range $\eta \in[1,5]$ and for atmospheric pressure.

As an example we consider the case when $\eta \approx 4.5$, which gives $R^{*} \sqrt{\omega} \approx 7$. Assuming the operating frequency to be $1 \mathrm{GHz}$, the corresponding radius is $R^{*} \approx 0.01 \mathrm{~cm}$. This is about 


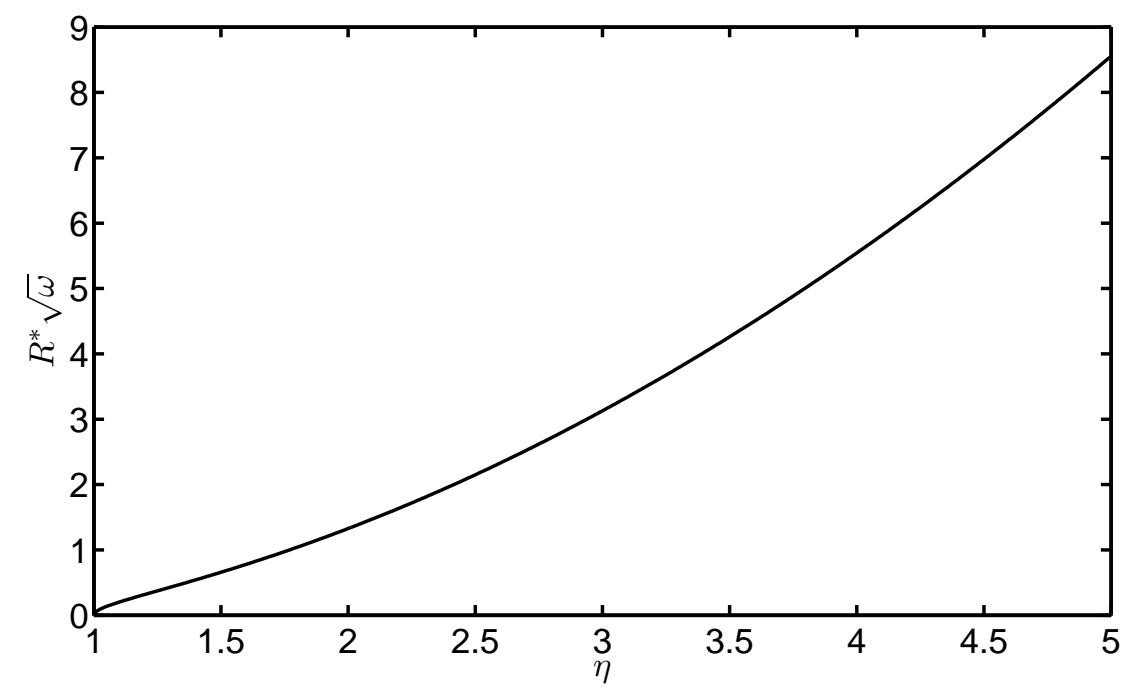

FIG. 6: Figure showing $R^{*} \sqrt{\omega}(\mathrm{m} / \sqrt{s})$ as a function of $\eta$ for Air at atmospheric pressure.

10 times larger than the attachment length, and the model should be valid. This means that if the plasma ball radius is less than $0.01 \mathrm{~cm}$, it will decay, whereas if it is larger, it will expand. At a frequency of $100 \mathrm{GHz}$, the critical radius becomes 10 times smaller, i.e. on the same scale as the attachment length, meaning that the model is unable to predict a safe radius, under which the plasma will disappear. It does however predict that any plasma ball with a radius much larger than this radius, and the attachment length, will expand indefinitely. If we lower the frequency to $100 \mathrm{kHz}$, the critical radius becomes a 100 times larger, i.e. $0.1 \mathrm{~cm}$. This clearly shows the physical relation between the frequency and the critical radius. When the frequency is lowered, the energy of the electric field goes down, and the heating of the plasma decreases, leading to a larger critical radius. Regarding the curve in Fig.6, it should be emphasized that the left part, close to $\eta=0$, which corresponds to very small values of $R^{*}$, is not reliable, since for any frequency, the critical radius will be smaller than the attachment length, and the model can not make reliable predictions in that range.

It is also of interest to examine the pressure and frequency dependance for the critical radius. Given that the ratio $\eta=E_{a 0} / E_{0}$ is kept constant, and using $E_{a 0} \propto p$, we get

$$
R^{*} \sqrt{\omega}=\frac{p_{0}}{p} R_{0}^{*} \sqrt{\omega_{0}}
$$

Thus, the critical radius decreases with increasing pressure, and increases with decreasing pressure. This can be intuitively understood, since the capacity of Air to absorb energy 
from the electrons should increase with increasing density, and the resulting increase in collision frequency. The above formula is very useful for rescaling the system parameters. For example, if the frequency is $1 \mathrm{GHz}$, and the pressure is lowered from 760 Torr (atmospheric pressure) to 76 Torr, the critical radius will grow to 10 times the original value, i.e. roughly $0.1 \mathrm{~cm}$. When using the rescaling formula above one should always be careful to stay within the limits of validity of the assumptions for the model. The attachment length, $L_{a}$, scales as $p^{-1}$, and if we lower the pressure to 76 Torr, the attachment length increases from $\sim 10^{-4}$ $\mathrm{cm}$ to $\sim 0.01 \mathrm{~cm}$. Thus, if the original critical radius is within the limits of the model, and only the pressure is rescaled, the new radius is also within the limits. however, if pressure and frequency are rescaled simultaneously, one must take into consideration the rescaling of the attachment length, and also keep in mind that the quasistatic approximation is only valid when $R^{*} \ll \lambda$.

Currently there are no experiments which can be immediately compared with the model and calculations above, but several similar situations have been studied. For example, an analysis has been made of the "role of absorbing inclusions in the optical breakdown in transparent media" ${ }^{11}$, where also a critical radius of the absorbing region was found, above which the breakdown region became unstable and changed character from local to global. However, this analysis did not involve the intricate nonlinear interplay between plasma formation, screening of microwave transmission and subsequent gas heating that is crucial in the present study. Also there are several experiments where the behavior of the breakdown dynamics was found to depend critically on the dimensions of the hot spot as well as on its temperature. Already in 1961, some relevant experimental observations were reported ${ }^{12}$. It was noted that the breakdown threshold depended on the absorption properties of the hot spot. In particular it was observed that global breakdown was only initiated when the absorption was strong enough to cause heating to orange or white temperatures. Regions heated to dull red temperatures could remain in the waveguide without developing to full scale breakdown and without causing harmful effects. The importance of gas heating in initiating a discharge has also been emphasized in the experimental works reported in ${ }^{13,14}$. In a recent paper particularly relevant for the present work $^{15}$, is presented a description of an experiment where the microwave field intensity was below the breakdown threshold, but nevertheless a discharge was initiated when the gas (Air) was heated locally by a laser spark. We emphasize that the size of the initially heated region in the experiment was very small 
with the diameter of the laser spark being only around a few micrometers.

\section{CONCLUSION}

An investigation has been made of the thermal stability properties of a breakdown plasma situated in a homogeneous electric rf field in a gas where attachment dominates the electron losses. The considered model is based on a spherical plasma in Air, but the results should apply to more arbitrary shapes and other attachment dominated gases. The thermal conduction equation for Air has been solved using an approximate expression for the heating power of the plasma due to absorption of the self consistent (i.e. suppressed by the dielectric properties of the plasma to obtain vanishing net ionization frequency) rf field in the plasma. This problem allows two equilibrium solutions, one stable, corresponding to the situation of no plasma, i.e. vanishing radius, and one unstable with a specific radius, $R^{*}$. For hot spots with an original radius larger than this radius, the subsequent breakdown plasma will monotonously expand, ultimately leading to global breakdown. Below this radius the plasma will shrink to ultimately disappear. The main aim of the present study is to highlight and understand the main physical effects involved in the interplay between the formation of a breakdown plasma and the further nonlinear dynamics where the electric field is suppressed by the dielectric shielding due to the plasma, the subsequent heating of the gas and the thermal dynamics of the gas. The result gives a valuable indication of the upper limit on the size of critically heated regions and regions of field enhancements above the local breakdown threshold for any particular system and shows that small scale hot spots may occur without neither developing to full scale breakdown nor affecting the over all performance of the device.

1 A. D. MacDonald, Microwave Breakdown in Gases, John Wiley and Sons, New York (1966)

2 Yu. P. Raizer, Gas Discharge Physics, Springer, Berlin (1991)

3 W. C. Taylor, W. E. Scharfman and T. Morita T 1971 Advances in Microwaves vol 7 ed L Young (New York: Academic)

4 U. Jordan, D. S. Dorozhkina, V. E. Semenov, T. Olsson, D. Anderson, M. Lisak, J. Puech, I. M. Nefedov and I. A. Shereshevskii, IEEE Trans. Plasma Science, 35, 542 (2007) 
${ }^{5}$ G. C. Light and E. C. Taylor, "Microwave breakdown in high-temperature Air", J. Appl. Phys. 39, $1591(1968)$

6 D. Anderson, M. Lisak and T. Lewin, "Thermal Lowering of the Threshold for Microwave Breakdown in Air-Filled Waveguides" IEEE MTT, 35, 653 (1987)

7 Said E El-Khamy, "The theory of transient microwave heating and ionization of low-pressure air", J. Phys. D: Appl. Phys., 7, 581 (1974)

8 J. D. Jackson, Classical Electrodynamics, John Wiley and Sons, New York, (1967)

9 N. A. Zykov, R. M. Sevast'yanov, and K. I. Voroshilova, "Transport properties of nitrogen, oxygen, carbon dioxide, and air at low densities and temperatures from 50 to $3000^{\circ} \mathrm{K}$ ", J. of Engineering Physics and Thermophysics, 43, 77 (1982)

10 J. T. Mayhan and R. V. DeVore, "Interaction of intense rf fields with heated Air", J. Appl. Phys. 39, 5746 (1968)

11 S. I. Anisimov and B. I. Makshantsev, "Role of absorbing inclusions in the optical breakdown in transparent media", Sov. Phys. Solid State 15, 743 (1973)

12 W. Beust and W. L. Ford, "Arcing in CW transmitters", Microwave Journal 10, 91 (1961)

13 Yu. Ya. Brodskii, S. V. Golubev, V. G. Zorin, A. G. Luchinin and V. E. Semenov, ”New mechanism of gas dynamic propagation of a discharge", Sov. Phys. JETP 57, 989 (1983)

14 N. A. Bogatov, Yu. V. Bykov, N. P. Venediktov, S. V. Golubev, V. G. Zorin, A. G. Eremeev and V. E. Semenov, "Gas dynamic propagation of a nonequilibrium microwave discharge", Sov. J. Plasma Phys. 12, 416 (1986)

15 S. A. Afanasiev, V. G. Broskii, Yu. F. Kolesnichenko "Ignition of UHF discharge using a laser spark", Pisma v Zhurnal Tekhicheskoi Fiziki 36, 73 (2010) (in Russian) 